\title{
Syringe Purification and HRP-Conjugation of Goat IgGs Used in Quality Control of Erythropoietin
}

\author{
Marcel Bassil (Corresponding author) \\ Biotechnology Department, Benta Pharma Industries \\ PO Box 70 31, Dbayeh, Zouk el Khrab 104, Lebanon \\ Tel: 961-7192-1665Ｅ-mail: marcel.bassil@bpi.com.lb
}

Nataliia Pavliuchenko

Biotechnology Department, Benta Pharma Industries

PO Box 70 31, Dbayeh, Zouk el Khrab 104, Lebanon

Tel: 961-7109-8979Ｅ-mail: nataliia.pavliuchenko@bpi.com.lb

\begin{abstract}
Elia Raya
Clinical Laboratory Sciences Program, Faculty of Health sciences, University of

Balamand, Beirut, Lebanon

E-mail: elia.raya@std.balamand.edu.lb
\end{abstract}

Received: March 13, 2018 Accepted: April 30, 2018 Published: May 3, 2018

Doi: 10.5296/jab.v6i2.12829 URL: http://doi.org/10.5296/jab.v6i2.12829

\begin{abstract}
Goat anti-rabbit IgGs were purified from serum using Protein $G$ affinity chromatography. The purity of eluted fractions was tested using SDS-PAGE, and the functionality of IgGs was tested in Western Blot analysis of Erythropoietin, after conjugation with Horseradish Peroxidase.
\end{abstract}

Keywords: Horseradish Peroxidase (HRP), IgG, Purification, Serum, Erythropoietin 


\section{Introduction}

Purification of antibodies from serum of blood is a technique used to isolate and purify specific antibodies from a large number of proteins including other types of antibodies present in serum. Several techniques can be implemented in serum purification including physicochemical fractionation, class-specific affinity, or antigen specific affinity. Class-specific affinity purification is the cheapest, fast and effective technique for purification of antibodies using such ligands as Protein A, G and L (Arora, Ayyar, \& O’Kennedy, 2014).

Protein $\mathrm{G}$ affinity chromatography is a technique used to separate IgGs from other proteins based on affinity. Protein $\mathrm{G}$ is a bacterial protein that is produced from group G Streptococci, it has high affinity and can efficiently bind to the Fc region of IgGs, and that is why protein $\mathrm{G}$ affinity chromatography is considered an effective method for the purification of goat IgGs (Page \& Thorpe, 2002). The obtained antibodies after purification can be used in immunological tests.

\section{Materials and Methods}

\subsection{Serum Used for Purification}

Lyophilized Goat Anti Rabbit $\operatorname{IgG}(\mathrm{H}+\mathrm{L})$ serum was purchased from Jackson Immunoresearch, USA.

\subsection{Resin for Purification}

For purification of goat IgGs used Pierce ${ }^{\mathrm{TM}}$ Chromatography Cartridges Protein G from Thermo Scientific.

\subsection{Dialysis}

To perform dialysis of purified antibodies used Micro Float-A-Lyzer Dialysis Device, 8-10 kDa MWCO from Spectrumlabs.

\subsection{HRP Conjugation Kit}

Conjugation of IgGs with HRP was performed using Lightning-Link ${ }^{\circledR}$ Horseradish Peroxidase (HRP) kit from Innova Biosciences Ltd.

\subsection{Western Blot and SDS-PAGE}

Western Blot analysis was done as described by (Mahmood \& Yang, 2012) with some modifications, using Trans-Blot ${ }^{\circledR}$ Turbo ${ }^{\text {TM }}$ Transfer System, Bio-Rad for semi-dry transfer of the proteins. All images were captured using VersaDoc Imaging System, Bio-Rad.

\section{Results}

\subsection{Purification and Determination of Concentration of IgGs}

Lyophilized Goat anti-rabbit antisera was rehydrated and diluted 5 times with binding buffer resulting in $31.5 \mathrm{mg}$ of total proteins. The solution was loaded on Protein G Chromatography Cartridge that was equilibrated with the same Binding buffer using a syringe procedure described in manufacturer's instructions. The resin was washed with Binding Buffer and this step was controlled using spectrophotometer. IgGs were eluted with Glycine buffer $\mathrm{pH}=2.5$ into $0.3 \mathrm{~mL}$ fractions with following $\mathrm{pH}$ adjustment with $1 \mathrm{M}$ Tris- $\mathrm{HCl} \mathrm{pH}=8.5$.

Absorbance of the collected fractions was measured by spectrophotometer at $280 \mathrm{~nm}$ using the elution buffer as a blank (Table 1). 6 fractions were collected with total yield of pure IgGs 
$10.5 \mathrm{mg}$.

Table 1. Absorbance and concentration of eluted fractions

\begin{tabular}{lllllll}
\hline $\begin{array}{l}\text { Elution } \\
\text { fraction } \mathrm{N}^{\circ}\end{array}$ & 1 & 2 & 3 & 4 & 5 & 6 \\
\hline $\begin{array}{l}\text { Absorbance } \\
\text { (at } 280 \mathrm{~nm})\end{array}$ & 0.48 & 1.0212 & 1.4057 & 1.0771 & 0.4010 & 0.8991 \\
$\begin{array}{l}\text { Concentration } \\
(\mathrm{mg} / \mathrm{mL})\end{array}$ & 0.369 & 7.855 & 21.62 & 8.28 & 3.08 & 0.69 \\
\hline
\end{tabular}

Concentration of eluted fractions was calculated using the formula:

$\mathrm{C}_{\mathrm{mg} / \mathrm{mL}}=\mathrm{Abs}_{280} / 1.3 \times$ dilution factor, where 1.3 (TECH TIP \#6 Extinction Coefficients Thermo Scientific) is extinction coefficient for goat IgG.

\subsection{SDS-PAGE Analysis of Collected Fractions}

SDS-PAGE in reducing conditions of purified antibodies $(\mathrm{Ab})$ using Coomassie staining showed 2 bands for each collected fraction that correspond to heavy and light chains of IgG (Figure 1).

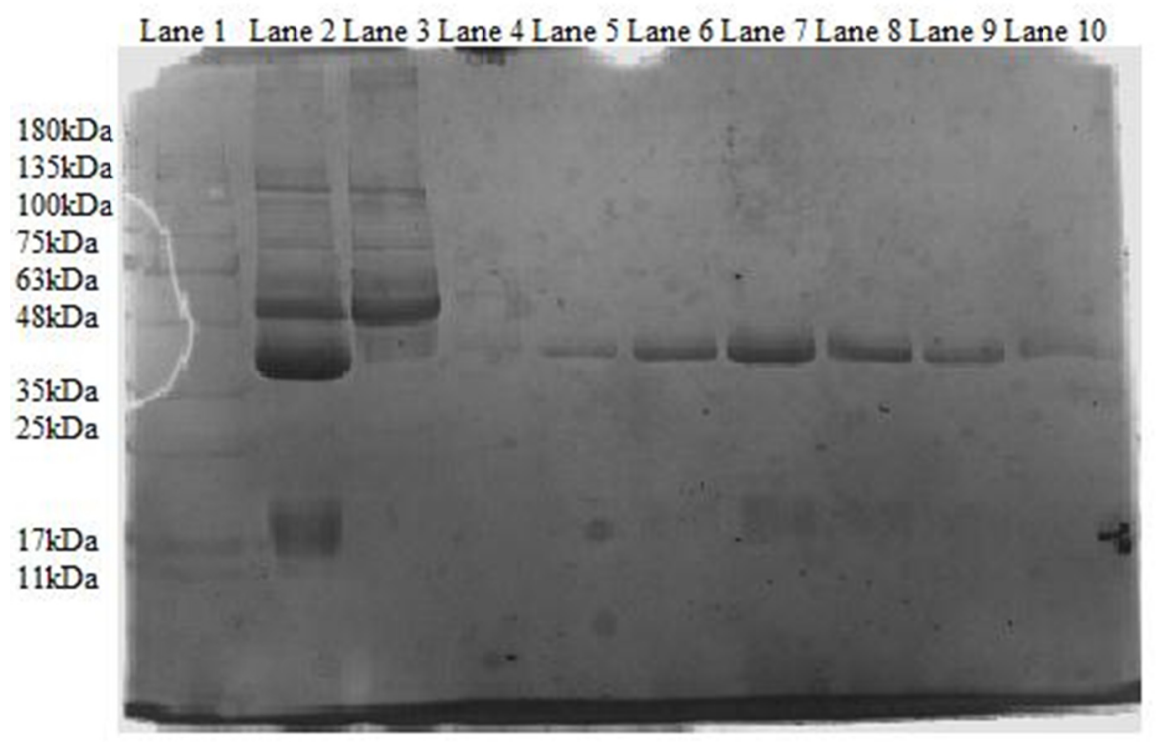

Figure 1. SDS-PAGE (10\%) of collected elution fractions. Lane 1: Ladder; Lane 2: serum; Lane 3: flowthrough 1; Lane 4: flowthrough 2; Lane 5: elution 1; Lane 6: elution 2; Lane 7: elution 3; Lane 8: elution 4; Lane 9: elution 5; Lane 10: elution 6

\subsection{Conjugation Procedure and SDS-PAGE Analysis of Obtained Conjugate}

The 2nd fraction was chosen for conjugation with Horseradish Peroxidase (HRP). Therefore dialysis of the sample $(150 \mu \mathrm{L})$ was performed overnight to exchange the buffer to $1 \times \mathrm{PBS}$ (phosphate buffered saline) that is compatible with HRP-conjugation kit. Retrieved antibody concentration was calculated by measuring $\mathrm{Abs}_{280}$, using 1.3 as extinction coefficient for goat 
IgG, and concentration was adjusted with $1 \times \mathrm{PBS}$ to $1 \mathrm{mg} / \mathrm{mL}$.

$100 \mu 1$ of goat anti-rabbit IgG with concentration of $1 \mathrm{mg} / \mathrm{mL}$ were taken for HRP conjugation. The HRP conjugation was performed overnight as per manufacturer's instructions using ratio $1: 4$, and resulted in $100 \mu 1$ of Goat anti-rabbit IgG HRP labeled. The conjugate was placed at $+4^{\circ} \mathrm{C}$. For control of conjugation, SDS-PAGE was done using samples of IgG before and after conjugation in reducing and non-reducing conditions (Figure 2).

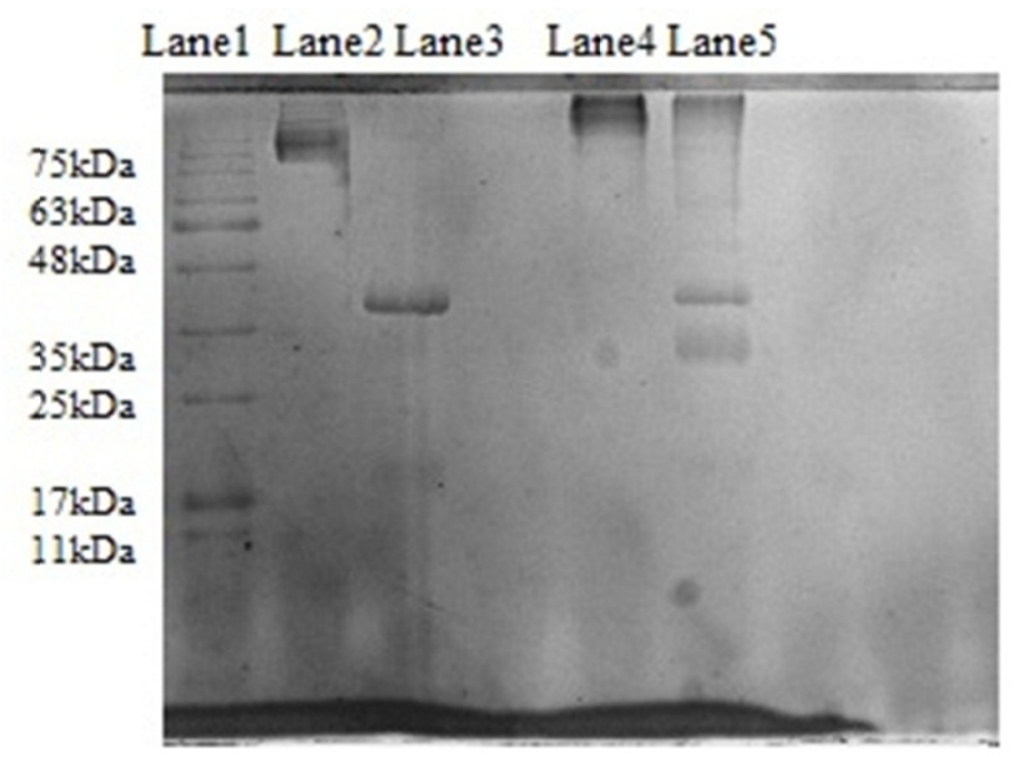

Figure 2. SDS-PAGE (10\%) analysis of dialyzed IgG and IgG-HRP conjugates. Lane 2: IgG dialyzed, non-reducing; Lane 3: IgG dialyzed, reducing; Lane 4: HRP conjugated IgG, non-reducing; Lane 5: HRP conjugated, IgG reducing

\subsection{Western Blot Analysis}

To test functionality of Ab-HRP conjugate, performed SDS-PAGE of Erythropoietin (reducing and non-reducing conditions) following by transfer of the protein. The transfer was controlled by staining the gel in Coomassie blue (Figure 4) and the nitrocellulose membrane in Red Ponceau (Figure 3). 


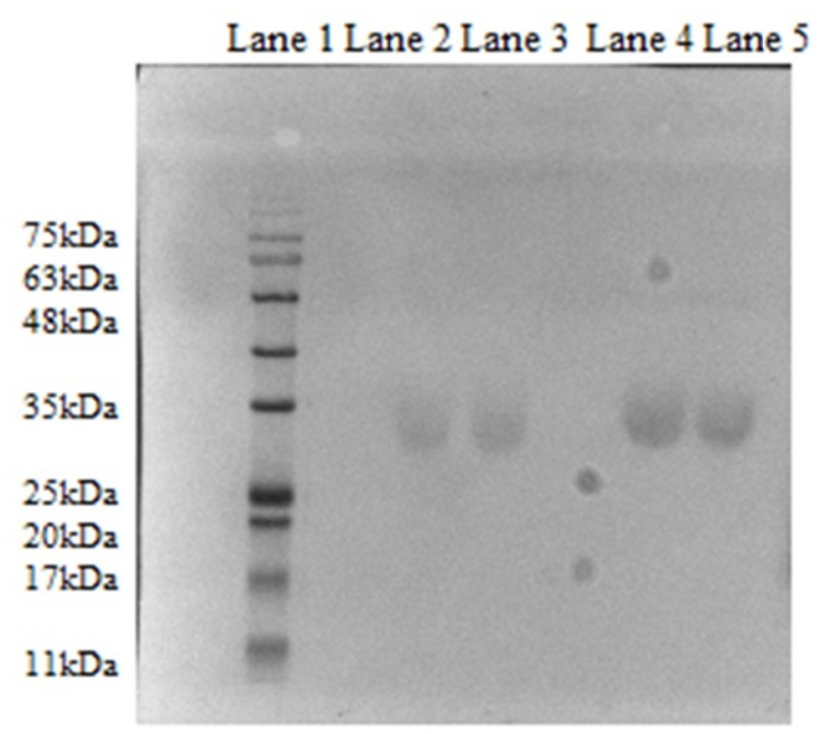

Figure 3. Ponceau Red staining of the nitrocellulose membrane after transfer. Lane 1: Erythropoietin reducing; Lane 2: Erythropoietin reducing; Lane 3: Erythropoietin non-reducing; Lane 4: Erythropoietin non-reducing.

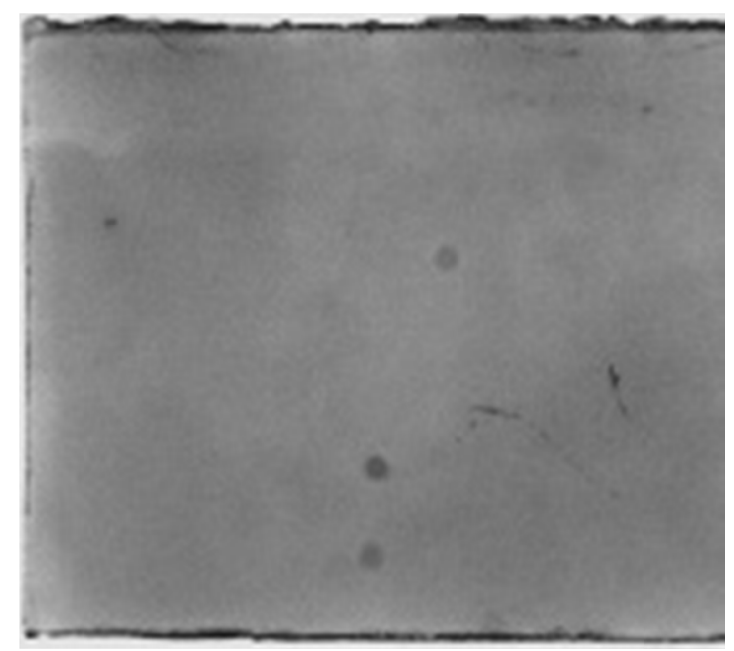

Figure 4. Coomassie Blue staining of the gel after transfer. No proteins are present

After overnight blocking in 5\% Bovine Serum Albumin, the membrane was incubated with primary Rabbit anti-Erythropoietin $\mathrm{Ab}$ at working dilution 1:5000 followed by incubation with secondary $\mathrm{Ab}$ (purified and HRP-conjugated in-house) at dilution 1:2 000. Between each step the nitrocellulose membrane was washed 3 times with $1 \times$ PBS-T (Tween).

3,3',5,5'-Tetramethylbenzidine (TMB) Liquid Substrate System for Membranes was used as substrate for HRP. Western blot analysis revealed a protein of a molecular weight equaling $30.5 \mathrm{kDa}$ that was detected in all four wells regardless of reducing or non-reducing environment (Figure 5). 


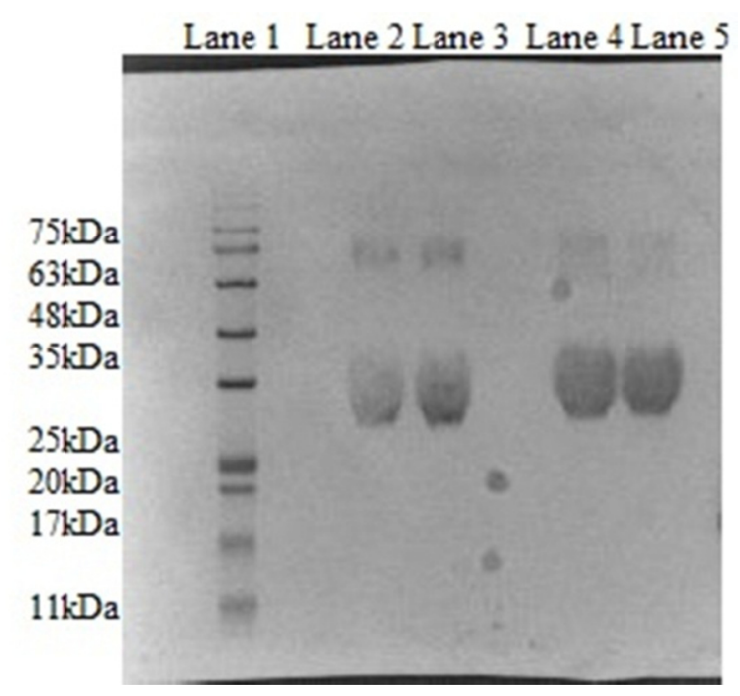

Figure 5. Nitrocellulose membrane after incubation with TMB. Lane 2: Erythropoietin reducing; Lane 2: Erythropoietin reducing; Lane 3: Erythropoietin non-reducing; Lane 4:

Erythropoietin non-reducing.

\section{Discussion}

Manual purification was performed using syringe procedure. During the purification procedure the washing step was controlled by measuring the absorbance at $280 \mathrm{~nm}$, to determine the levels of unbound proteins still present in the column.

Results obtained from the SDS-PAGE electrophoresis show highly concentrated amount of proteins in the wells containing serum, and flowthrough 1 . However, only 2 bands were found in the elution fractions; due to reducing conditions, disulfide bonds are broken which implies that the two bands observed correspond to the light and heavy chains of IgGs with the heavy chain having a higher molecular weight than the light chain. The fact that only two bands can be seen on the gel validates that the purification process was successful since the only protein present in the eluted fractions was IgG. No other proteins were bound or eluted along with the IgGs.

The dialyzed IgGs show 1 thick band corresponding to IgGs in non-reducing conditions whereas in reducing conditions 2 bands can be seen indicating the separation of the light and heavy chain. Concerning the HRP conjugated IgG samples, in non-reducing conditions, a thick band can be visualized indicating that a protein band with a molecular weight different from that of IgG was detected, whereas in reducing conditions, 3 bands can be visualized. The highest and the lowest molecular weight bands out of the three correspond to both heavy and light chain respectively. The third band is the HRP which dissociated from the IgG and was detected on its own. These results indicate that all IgGs were bound to the HRP forming IgG-HRP complex and that the conjugation procedure was successful.

Western blot analysis revealed a protein of a molecular weight equaling $30.5 \mathrm{kDa}$ which was detected in all four wells regardless of reducing or non-reducing environment. This signifies that the purified secondary antibodies used for the western blot did in fact bind to the primary antibodies and were detected through western blot. This leads us to the conclusion that the 
purified antibodies retrieved are functional and can be used as secondary antibodies in immunological tests.

Moreover, western blot analysis done with the same amounts of protein using commercial goat anti-rabbit secondary antibodies HRP-conjugated (Figure 6) shows highly similar results to the ones obtained using in-house purified and conjugated secondary goat anti-rabbit antibodies. This further proves the functionality of the obtained purified antibodies in comparison to the commercial ones.

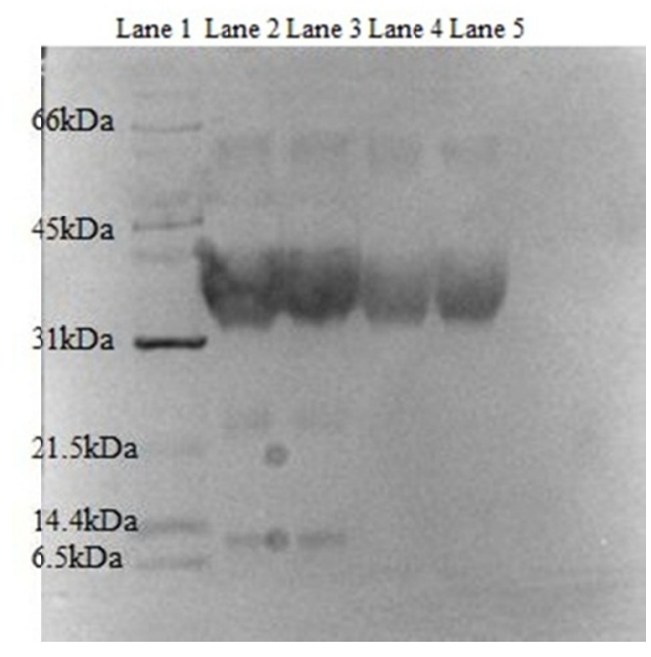

Figure 6. Nitrocellulose membrane after blotting (secondary Ab goat anti-rabbit commercial). Lane1: Marker (1:20 with reducing LSB); Lane 2: Reducing Sample (10 $\mathrm{g} / \mathrm{well})$; Lane 3:

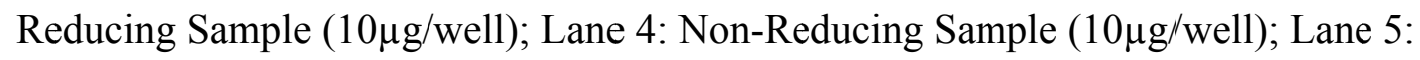
Non-Reducing Sample (10 $\mu \mathrm{g} / \mathrm{well})$.

\section{Conclusion}

Purification using Protein G Chromatography cartridges led to the retrieval of pure IgGs, which was corroborated by SDS-PAGE. The purified antibodies were also functional which is shown clearly by the results of the SDS-PAGE of the HRP-conjugated IgGs and the detection of Erythropoietin in Western blot analysis. Therefore, we can conclude that this protocol of purifying antibodies from serum is an effective way of obtaining secondlary antibodies that are pure and functional. This will provide the laboratory with a cheap and more abundant source of secondary antibodies to be used in Quality Control immunological techniques.

\section{Acknowledgement and Sponsoring information}

The research was financed by the Biotechnology department at Benta Pharma Industries

\section{References}

Arora, S., Ayyar, B. V., \& O’Kennedy, R. (2014). Affinity Chromatography for Antibody Purification. In: Labrou N. (eds) Protein Downstream Processing. Methods in Molecular Biology (Methods and Protocols), vol 1129. Humana Press, Totowa, NJ. https://doi.org/10.1007/978-1-62703-977-2_35

Page, M., \& Thorpe, R. (2002). Purification of IgG Using Protein A or Protein G. In J. M. Walker (Eds) The Protein Protocols Handbook. Humana Press. 
https://doi.org/10.1385/1-59259-169-8:993

2018, Vol. 6, No. 2

Mahmood, T., \& Yang, P.-C. (2012). Western Blot: Technique, Theory, and Trouble Shooting. North American Journal of Medical Sciences, 4(9), 429-434. http://doi.org/10.4103/1947-2714.100998

TECH TIP \#6 Extinction Coefficients Thermo Scientific

\section{Glossary}

HRP - Horseradish Peroxidase

PBS - phosphate buffered saline

PBS-T - phosphate buffered saline tween

$\mathrm{Ab}$ - antibodies

IgG - Immunoglobulin G

SDS-PAGE - sodium dodecyl sulfate polyacrylamide gel electrophoresis

kDA - kilodalton

MWCO - molecular weight cut off

Abs - Absorbance

\section{Copyright Disclaimer}

Copyright reserved by the author(s).

This article is an open-access article distributed under the terms and conditions of the Creative Commons Attribution license (http://creativecommons.org/licenses/by/3.0/). 